\title{
Laboratory Methodologic Approach in Prolonged Activated Partial Thromboplastin Time Test \\ Guido D'Angelo ${ }^{1 *}$
}

1. Former executive Laboratory of Clinical-Chemistry, Hematology and Microbiology, ASST Valle Olona, Gallarate Hospital, Via Pastori 4, 21013, Gallarate, Varese, Italy.

\begin{tabular}{c} 
KEYWORDS \\
\hline Coagulation; \\
aPTT; \\
Bleeding; \\
Laboratory approach; \\
Acquired hemophilia A \\
\hline Article Info \\
\hline Received 2019/02/23; \\
Accepted 2019/03/05; \\
Published Online 2020
\end{tabular}

\begin{abstract}
The pathological activated partial thromboplastin time test is a data that we can find in all clinical laboratories routinely. The hemato-coagulative clinical case here reported aims to point out how the laboratory can provide a correct diagnosis by methodological rational setting and, consequently, carry out an appropriate therapy, or reassure the patient that the pathological data will not cause bleeding, even in case of surgery.
\end{abstract}

Corresponding Information: Guido D'Angelo PhD, Former executive Laboratory of Clinical-Chemistry, Hematology and Microbiology, ASST Valle Olona, Gallarate Hospital, Via Pastori 4, 21013, Gallarate, Varese, Italy. Email: danguido@ libero.it. Tel: +39 0331.751.456

Copyright $\odot 2020$. This is an open-access article distributed under the terms of the Creative Commons Attribution-noncommercial 4.0 International License which permits copy and redistribute the material just in noncommercial usages, provided the original work is properly cited.

\section{Introduction}

The time necessary for the clot fibrin formation when the citrate plasma in presence of an activator, (e.g. kaolin, celite, ellagic acid, micronized silica) and phospholipids (e.g. cephalin), is recalcified and characterizes activated partial thromboplastin time (aPTT) test expression (1).

The test is affected by the activity of coagulation factors I (fibrinogen), II (prothrombin), V, VIII, IX, X, $\mathrm{XI}$ and XII. When one of the coagulation factors is deficient, such as in A and B hemophilia or in the consumption coagulopathy, the aPTT is prolonged (2).

aPTT test is sensitive in defects of the intrinsic and common pathway coagulation system, particularly in FVIII deficiency (3). Moreover, it is sensitive to the deficiency of some factors involved in the intrinsic system contact phase, such as FXII, prekallikrein (PK) and high molecular weight kininogen (HMWK) (4).

The test is also sensitive to the action of circulating anticoagulants, "lupus-like", which interferes with phospholipid dependent laboratory test of coagulation (5). This inhibitory action on the intrinsic coagulation system triggers a thrombophilic condition.
Before undertaking a more detailed investigation, it's important to rule out artifactual causes of an abnormal aPTT such as a high hematocrit value, lipemic, hemolyzed (6), or icteric plasma, delays, extreme temperatures and any other causes that underly an incorrect pre-analytic approach that the laboratory must carefully identify (7).

aPTT test is the reference laboratory test for monitoring unfractionated heparin therapy.

\section{Case Report}

A 71-year-old woman contacts the attending physician for the occurrence of recurrent episodes of bruising and hematomas located in the upper and lower limbs.

Outpatient laboratory tests highlighted a normochromic and normocytic anemia, white blood cells (WBC) count, differential white blood cell (DWBC) count and platelets (PLTs) count were normal by number and morphology.

The coagulative tests showed an aPTT test prolonged $(\mathrm{aPTT}=55.7 \mathrm{~s}-$ laboratory $\mathrm{r}$. v. $=25-36 \mathrm{~s})$, while the Prothrombin Time (PT) test was normal (PT $=9.0 \mathrm{~s}-$ laboratory $\mathrm{r}$. v. $=10-13 \mathrm{~s})$. 
The patient reported a clinical history of arterial hypertension. She does not have a history of past cutaneous and/or mucous bleeding. She does not take drugs that could cause diathesis bleeding. A family history for hemorrhagic diseases was not reported.

After hematological consultation, hospitalization was planned. During hospitalization, the hemorrhagic diathesis persisted, so much that the patient was also subjected to blood transfusions for the presence of severe anemia (Hemoglobin $(\mathrm{Hb})<70 \mathrm{~g} / \mathrm{L})$. In Table 1 the main laboratory routine tests are reported. Other routine tests were normal.

Table 1. Patient's blood tests values during hospitalization

\begin{tabular}{|c|c|c|}
\hline Test & Values found & $\begin{array}{c}\text { Laboratory reference } \\
\text { value }\end{array}$ \\
\hline PT & $9.0 \mathrm{~s}$ & $10-13 \mathrm{~s}$ \\
\hline aPTT & $59.2 \mathrm{~s}$ & $25-36 \mathrm{~s}$ \\
\hline Fibrinogen & $680 \mathrm{mg} / \mathrm{dL}$ & $130-330 \mathrm{mg} / \mathrm{dL}$ \\
\hline $\mathbf{H b}$ & $65 \mathrm{~g} / \mathrm{L}$ & $120-150 \mathrm{~g} / \mathrm{L}$ \\
\hline $\mathbf{H t}$ & $23.8 \%$ & $36-44 \%$ \\
\hline $\mathbf{W B C}$ & $12 \times 10^{9} / \mathrm{L}$ & $4.0-10.0 \times 10^{9} / \mathrm{L}$ \\
\hline PLT & $476 \times 10^{9} / \mathrm{L}$ & $150-450 \times 10^{9} / \mathrm{L}$ \\
\hline
\end{tabular}

\section{What should the laboratory do in case of pathological aPTT?}

In isolated prolonged aPTT, one of the first evaluations is to consider the fibrinogen test. If its value is $<100 \mathrm{mg} / \mathrm{dL}$, the data would address for quantitative alterations, resulting in a hypofibrinogenemia and consequently prolonged aPTT (Table 2). Fibrinogen test, although simple and not expensive, can be inaccurate in case of disseminated intravascular coagulation, liver disease, kidney disease, dysfibrinogenemia due to thrombolytic therapy, or increased fibrinogen concentration. The fibrinogen dosage must be performed according to the Clauss method (8).

Table 2. Main causes of hypofibrinogenemia

Hereditary afibrinogenemia

Dysfibrinogenemia

Embolism

Hemophilia

Disseminated intravascular coagulation

Fibrinolysis

Eclampsia

Liver disease

Tumors

Thrombin Time (TT) is another test that must be considered if aPTT is prolonged. The test measures the conversion time of fibrinogen into polymerized fibrin (8). The test is pathological in hypo and dysfibrinogenemia, in presence of fibrin and/or fibrinogen degradation products that influence the fibrin polymerization (e.g. paraproteinemia in myeloma), as well as in patients on heparin therapy or heparin sample contamination (e.g. flushing in central venous catheter (CVC) and/or accidental sample collection from the polluted line) (7). The abnormal TT test is corrected in patients with CVC and suspected sample heparin contamination, by the addition of heparin neutralizing agents (e.g. hepzyme or protamine sulfate). In these cases, also the normal reptilase time test indicates the polluted sample.

\section{Plasma "Mix Test" study}

The test consists of mixing in equal parts (50:50) the pathological specimen plasma with normal plasma; the latter must be obtained from a pool of normal plasmas that have no deficiency of coagulation factors (9). On the obtained mixture, the aPTT test is performed again.

Table 3 shows the procedure, the patient's baseline aPTT value, the patient's aPTT values on mix plasma and, finally, the values obtained after comparison $v s$ control plasma.

\begin{tabular}{|c|c|c|}
\hline $\begin{array}{c}\text { aPTT } \\
\text { Baseline }\end{array}$ & $\begin{array}{c}\text { aPTT } \\
\text { Plasma Mix 50/50 } \\
\text { Incubation 30min } \\
\text { at room temperature }\end{array}$ & $\begin{array}{c}\text { aPTT } \\
\text { Plasma Mix } 50 / 50 \\
\text { Incubation } 2 \mathrm{~h} \text { at } 37^{\circ} \mathrm{C} \\
\text { Comparison with control plasma }\end{array}$ \\
\hline $\begin{array}{c}59.2 \mathrm{~s} \\
\text { Ratio }=2.15\end{array}$ & $\begin{array}{c}\text { aPTT Mix }=58.7 \mathrm{~s} \\
v s \\
\text { Normal Pool Plasma } \\
=28.0 \mathrm{~s}(\text { Ratio }=2.13)\end{array}$ & $\begin{array}{c}\text { aPTT Mix }=58.4 \mathrm{~s} \\
\qquad s \\
\text { Control Plasma }=27.5 \mathrm{~s} \\
\quad(\text { Ratio }=2.12)\end{array}$ \\
\hline \multicolumn{3}{|c|}{$\begin{array}{l}\text { aPTT Control Plasma }=27.5 s(r . v .90 \%=23.0 s-31.9 s) \\
\text { aPTT Normal Pool Plasma }=28.0 s-\text { Ratio }=1.0 \\
\text { Kit employed Actin FS }\left(\text { Siemens }{ }^{\circledast}\right) ; \text { activator }=\text { Ellagic acid }\end{array}$} \\
\hline
\end{tabular}

The suspicion of phospholipid inhibitors that can induce eventual thrombosis is justified if, on mixed plasma, the aPTT test is still pathological and a concomitant thrombocytopenia is eventually present $(10,11)$. If the anti-phospholipid dependent antibodies are negative, the dosage on both coagulation factors and specific inhibitors against coagulation factors is justified (3). Specific anticoagulation factor antibodies are mainly directed against FVIII causing profuse bleeding.

\section{What diagnosis was made?}

Summing up, the laboratory excluded the nonappropriateness of the sample and any hypodysfibrinogenemia.

The laboratory excluded the PK or HMWK deficiencies, also based on the clinical symptomatology characterized by profuse bleeding. 
Lupus anticoagulant antibodies presence were excluded by employing LAC, IgG and IgM anticardiolipin antibodies as well as IgG and IgM anti- $\beta 2$ glycoprotein 1 antibodies, and procoagulant factor deficiencies tests.

Regarding the mix test, the laboratory analyzed the comparison between seconds and ratio, as well as the Rosner index and the percentage correction, whose results turned out to be

$<5 \mathrm{~s},>1.2,>15 \%,<58 \%$, respectively (12-14).

Based mainly on mix test analysis, the next diagnostic step was both the dosage of coagulation factors and the specific inhibitors detection against coagulation factors, principally FVIII. The most important diagnostic data was the marked FVIII reduction, if not almost absent, due to the presence of marked inhibitor activity (Table 4).
Table 3. Main causes of hypofibrinogenemia

\begin{tabular}{|c|c|}
\hline FVIII $<1 \%$ & $\begin{array}{l}\text { Anti-FVIII inhibitor } \\
\text { Bethesda Unit }(\mathrm{BU})=\mathbf{2 5 0}\end{array}$ \\
\hline \multicolumn{2}{|r|}{ FIX $=57 \%$} \\
\hline \multicolumn{2}{|r|}{$\mathrm{FXI}=41 \%$} \\
\hline \multicolumn{2}{|r|}{ FXII $=48 \%$} \\
\hline $\begin{array}{l}\text { *One Bethesda } \\
\text { inactivates } 50 \% \\
\text { Cfor two hours }\end{array}$ & $\begin{array}{l}\text { is the amount of inhibitor that } \\
\text { normal plasma after incubation at } 37^{\circ}\end{array}$ \\
\hline
\end{tabular}

From the analysis results, the laboratory made the diagnosis of "acquired hemophilia $\mathrm{A}$ " due to the presence of anti-FVIII antibodies (15). Figure 1 shows the diagnostic pathway carried out by the laboratory after excluding a hypo or dysfibrinogenemia.

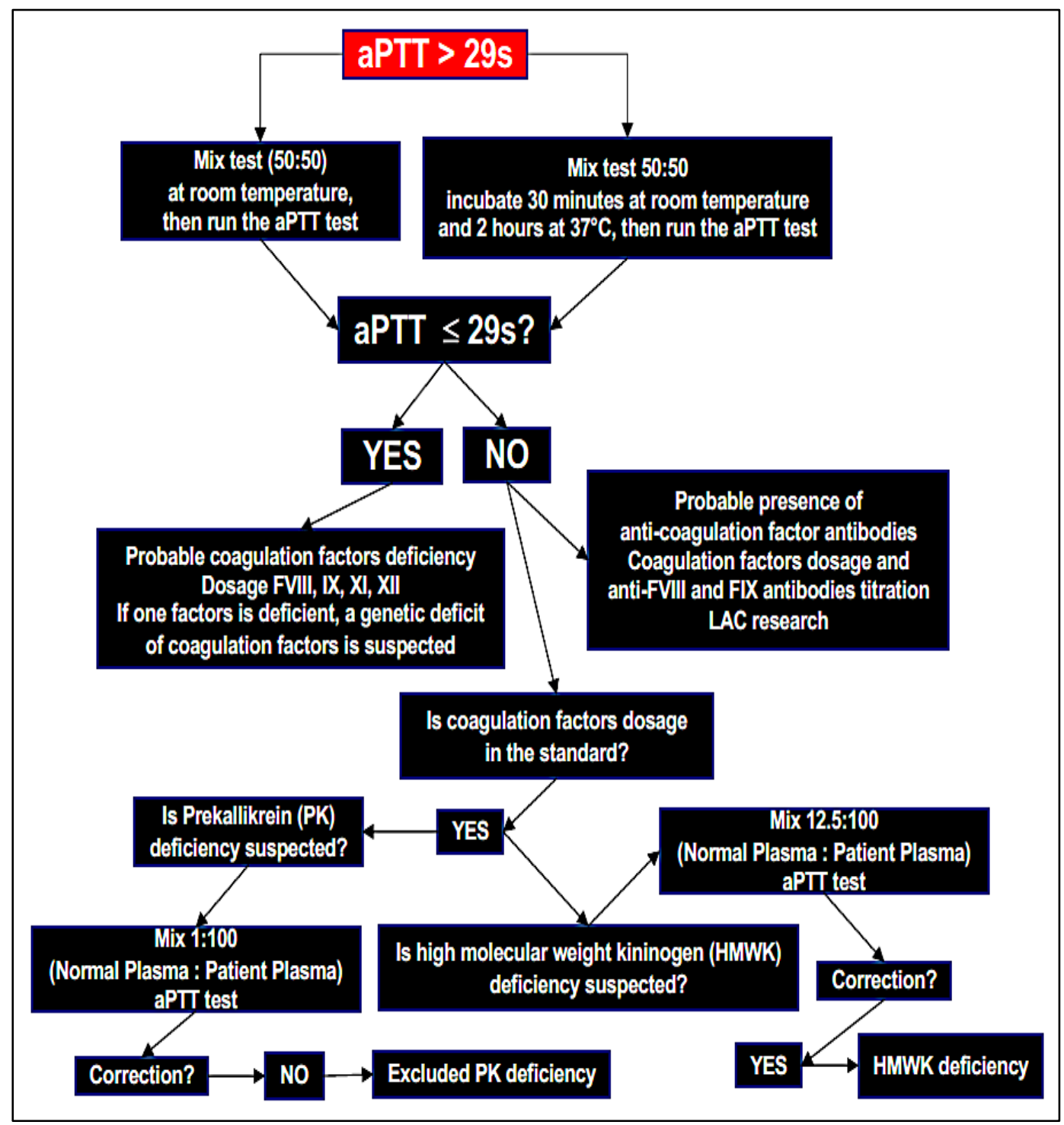

Fig. 1. Diagnostic proceedings in abnormal aPTT 
86 Laboratory Methodologic Approach in aPTT ....

Table 5. Conditions with prolonged aPTT without hemorrhagic diathesis

\begin{tabular}{|l|}
\hline Deficiency of: \\
\hline FXII \\
\hline High molecular weight kininogen (HMWK) \\
\hline Prekallikrein (PK) \\
\hline Lupus Anticoagulant (LAC) \\
\hline Sample anticoagulant excess (citrate) \\
\hline
\end{tabular}

Table 6. Main causes of acquired Hemophilia A and relational percentages

\begin{tabular}{|l|l|}
\hline \multicolumn{1}{|c|}{ Autoimmune diseases } & \\
\hline SLE, Rheumatoid Arthritis, Sjogren Synmdrome, Multiple Sclerosis, Myasthenia Gravis, \\
AHA, Graves-Basedow Disease \\
\hline Solid Tumors \\
\hline Prostate, Lung, Colon, Pancreas, Stomach, Head-Neck, Cervix, Breast \\
\hline Oncohematological diseases & \\
\hline CLL, NHL, MM, MDS, IM & \\
\hline Drugs & \\
\hline Penicillin, sulfamides, phenytoin, methyldopa, chloramphenicol & \\
\hline Pregnancy and postpartum & \\
\hline Idiopathic & $\mathbf{1 0 \%}$ \\
\hline
\end{tabular}

AHA = Autoimmune Hemolytic Anemia; SLE = Systemic Lupus Erythematosus; $C L L$ = Chronic Lymphocytic Leukemia; $N H L=$ Non-Hodgkin Lymphoma; MM = Multiple Myeloma; MDS = Myelodysplastic Syndrome; IM = Idiopathic Myelofibrosis

\section{Discussion}

In patients with bleeding diathesis, an accurate clinical history for previous bleeding, as well as family history, is mandatory, even in case of previous surgeries or changes in hemostatic status (16). The resulting reports are very important to identify patients who need further and more detailed investigation, since a prolonged aPTT, although pathological in some cases, may not cause hemorrhagic diathesis (Table 5) (17).

In acquired hemophilia A, FVIII inhibitory antibodies are immunoglobulins consisting mainly of heavy chain G4 class and $\kappa$ light chain; their action is directed against FVIII procoagulant activity (18). Regarding the functional activity, the kinetic inactivation by anti-FVIII antibodies can be type 1 or 2 . According to a linear kinetics, type 1 inhibitory antibodies inhibit the FVIII procoagulant activity completely. Type 2 inhibitory antibodies follow an exponential complex kinetics and are not able to inhibit FVIII procoagulant activity completely. Therefore, the Mix test must be performed and interpreted correctly. A short incubation may not show both the presence of type 2 inhibitors, so called "slow inhibitors" which present a complex kinetics (time-dependent kinetics), and low-title inhibitors that show a sensitivity lower than the method used.

In our case, the very significant FVIII deficiency justified the pathological laboratory data (prolonged aPTT), as well as the profuse clinical symptomatology characterized by bleeding with severe anemia.

Acquired hemophilia A has an annual incidence of 1-2 cases per million of inhabitants. In some carriers, bleeding can also be fatal. Major bleeding occurs in $80-90 \%$ of cases and mortality is significant, the range is between $10 \%$ and $22 \%$.

The causes that can determine the pathology are different; $50 \%$ of cases have an idiopathic etiology (Table 6).

The therapeutic approach is mainly applied to treat the hemorrhagic episode in case of emergency, as well as to prevent significant bleeding in surgery. A possible therapeutic association with immunomodulant drugs to eliminate the cellular clone responsible for self-antibody synthesis is also considered.

To monitor anti-hemorrhagic treatment, Factor VIII levels and/or inhibitor titers do not have a therapeutic indication. It is important to emphasize that in about $30 \%$ of cases the autoantibody can disappear spontaneously. 
The case reported underlines how, through a correct methodological approach, the laboratory can and must provide a correct diagnosis. A pathological aPTT may underline not only hemorrhagic problems but also a prothrombotic condition, two possibilities that impose different therapeutic approaches. Furthermore, although the aPTT test is abnormal, the rational methodological approach can rule out the possibility of bleeding even in case of surgery.

\section{Conflicts of interest}

The authors state no conflicts of interest in this study.

\section{References}

1. Mann KG. Biochemistry and physiology of blood coagulation. Thromb Haemost. 1999; 82: 165-174.

2. Lippi G, Favaloro EJ. Laboratory hemostasis: from biology to the bench. Clin Chem Lab Med 2018; 56: 1035-1045.

3. Detecting and Evaluating Coagulation Inhibitors and Factor Deficiencies by Media Lab, Inc. (LabCE) ASCLS P.A.C.E. Provider \#578 \& Florida Board of Clinical Laboratory Personnel Provider \#5010293.

4. Pancione Y, Fumi M, Sale S, Rocco V. Lack of high molecular weight kininogen and hemostasis global tests: a case Report. It J Lab Med 2013; 9: 166-169.

5. Rosner E, Pauzner R, Lusky A, Modan M, Many A. Detection and quantitative evaluation of lupus circulating anticoagulant activity. Thromb Haemost 1987; 57: 144-147.

6. D'Angelo G, Villa C, Tamborini A, Villa S. Evaluation of the main coagulation tests in the presence of hemolysis in healthy subjects and patients on oral anticoagulant therapy. Int J Lab Hemat 2015; 37: 819833.

7. Lippi G, Favaloro EJ. Preanalytical issues in hemostasis and thrombosis testing. Methods Mol Biol 2017; 1646: 29-42.

8. Laffan M, Manning R. Investigation of haemostasis. In: Lewis SM, Bain BJ, Bates I, editors. Dacie and Lewis Practical Haematology. 11th ed. London: Churchill Livingstone Elsevier. 2012. p. 423.
9. Kershaw G, Orellana D. Mixing tests: diagnostic aides in the investigation of prolonged prothrombin times and activated partial thromboplastin times. Semin Thromb Hemost 2013; 39: 283-290.

10. Devreese KM. Interpretation of normal plasma mixing studies in the laboratory diagnosis of lupus anticoagulants. Thromb Res. 2007; 119: 369-376.

11. Lupus anticoagulants, antiphospholipid antibodies, and antiphospholipid Syndrome chapter 39; by William L.Nichols, Kandice Kottke -Marchant in Laboratory Hematology Practice, first edition by Kandice Kottke-Marchant, Bruce H. Davis. 2012: 509525.

12. Baig MA, Sirasagi AK. Comparative evaluation of Rosner's index (ICA) Vs Chang's (\% correction) as a screening test (mixing study). J Diagn Pathol Oncol. 2018; 4: 196-201.

13. Chang SH, Tillema V, Scherr DA. A "percent correction" formula for evaluation of mixing studies. Am J Clin Pathol 2002; 117: 62-73.

14. Depreter B, Devreese KMJ. Differences in lupus anticoagulant final conclusion through clotting time or Rosner index for mixing test interpretation. Clinical Chemistry and Laboratory Medicine 2016; 54: 15111516, ISSN (Online) 1437-4331, ISSN (Print) 14346621.

15. Kruse-Jarres R, Kempton CL, Baudo F, et al. Acquired hemophilia A: Updated review of evidence and treatment guidance. Am J Hematol. 2017; 92: 695-705

16. Rehman HU. Elevated APTT? How best to follow-up. The Journal of Family Practice 2013; 62: E1-E3.

17. Patel N, Conley GW, McElroy LA, Refaai MA. Isolated prolonged activated partial thromboplastin time and contact factor deficiencies: case series and management review. Anesthesiol Open J. 2016; 1: 1923.

18. Ma AD, Carrizosa D. Acquired factor VIII inhibitors: pathophysiology and treatment. Hematol Am Soc Hematol Educ Program. 2006; 432-437.

\section{How to Cite This Article:}

D'Angelo G. Laboratory Methodologic Approach in Prolonged Activated Partial Thromboplastin Time Test. Mod Med Lab J. $2020 ; 3$ (2):83-87 